\title{
Spiky Strings in $\mathrm{AdS}_{4} \times \mathrm{CP}^{3}$ with Neveu-Schwarz Flux
}

\author{
Sachin Jain ${ }^{a}$ and Kamal L. Panigrahi ${ }^{b}$ \\ ${ }^{a}$ Institute Of Physics, Bhubaneswar, 751 005, India \\ ${ }^{b}$ Department of Physics, Indian Institute of Technology Guwahati, \\ Guwahati-781 039, India \\ E-mail: sachjain@iopb.res.in, panigrahi@iitg.ernet.in
}

\begin{abstract}
We study general rotating string solution in the $A d S_{4} \times \mathbf{C P}^{3}$ background with a $B_{N S}$ holonomy turned on over $\mathbf{C P}^{1} \subset \mathbf{C P}^{3}$. We find the giant magnon and single spike solutions for the string moving in this background corresponding to open spin chain. We calculate the corresponding dispersion relation among various conserved charges for both the cases. We further study the finite size effect on both the giant magnon and single spike solutions.
\end{abstract}

KEywords: AdS-CFT Correspondence. 


\section{Contents}

1. Introduction 1

2. Rotating String Solutions in ABJ theory 2

3. Giant Magnon and Single Spike Solutions

4. Finite Size effects 6

5. Conclusions 8

\section{Introduction}

Recently Aharony, Bergman, Jafferis and Maldacena (ABJM) [1] proposed a new class of gauge theory-string theory duality between $\mathcal{N}=6$ Chern-Simons (CS) theory and type IIA string theory on $\mathrm{AdS}_{4} \times \mathbf{C P}^{3}$. Based on this Aharony, Bergman, and Jafferis (ABJ) [2] identified further a class of $A d S_{4} / C F T_{3}$ duality with extended supersymmetry namely, a three dimensional $\mathcal{N}=6$ superconformal Chen-Simons theory with a gauge group $U(M)_{k} \times \overline{U(N)}_{-k}$, with $k$ being the level of the CS theory, is dual to type IIA string theory on $\mathrm{AdS}_{4} \times \mathbf{C P}^{3}$ with $\mathrm{B}_{N S}$ holonomy turned on over $\mathbf{C P} \mathbf{P}^{1} \subset \mathbf{C P}^{3}$.

In proving AdS/CFT duality [3], the integrability of both the string theory and the gauge theories have played a key role. The semiclassical string states in the gravity side has been used to look for suitable gauge theory operators on the boundary, in establishing the duality. In this connection, Hofman and Maldacena (HM) « considered a special limit where the problem of determining the spectrum of both sides becomes rather simple. The spectrum consists of an elementary excitation known as magnon which propagates with a conserved momentum $p$ along the spin chain. Further, a general class of rotating string solution in $\mathrm{AdS}_{5}$ is the spiky string [5, 6] which describes the higher twist operators from dual field theory view point. Giant magnons can be thought of as a special limit of such spiky strings with shorter wavelength. Recently there has been a lot of work devoted for the understanding of the giant magnon and spiky string solutions in various backgrounds see for example [7]-[27].There has also been numerous papers devoted for understanding the finite size corrections on these solutions [28].

In the present paper we study a general class of rotating string solution in the ABJ model. It has already been established that like its regular counter part, ABJ theory has an integrability structure in planar limit. In fact in 24] it has further been analyzed that the magnon dispersion relation remains exactly the same as that of ABJM, even the ABJ theory has an extra NS B field in its spectrum, thus showing no parity symmetry 
breaking effect. In this paper we study the giant magnon and spike solutions for strings corresponding to open spin chain. We concentrate on a particular sector of the theory which is the diagonal $S U(2)$ subgroup inside $\mathbf{C P}^{3}$, and study a general class of rotating strings. We solve the equations of motion and the Virasoro constraints for the Polyakov action of the string in the presence of a NS-NS $B$ field. We find out the general form of all the conserved charges and choose particular parametrization corresponding to a special relation among those charges. We further find the corresponding dispersion relation for the giant magnon and single spike solutions for the string moving in the $S U(2)$ subsector inside $\mathbf{C P}^{3}$. Finally, we study the finite size corrections for both the giant magnon and for the single spike solutions.

The rest of the paper is organized as follows. In section 2, we consider a rotating string solution in $R \times S^{2}$ with NS-NS $B$ field. We write down all the equations of motion, and Virasoro constraints for the string moving in this background. In section 3, we find the giant magnon and single spike solutions for the string in infinite size limit and the corresponding dispersion relations inside a particular parameter space. In section 4, we investigate the finite size effects for both the giant magnon and single spike solution. In section 5, we present our conclusions.

\section{Rotating String Solutions in ABJ theory}

We start by writing down the supergravity dual background of the ABJ theory

$$
\begin{aligned}
d s^{2}=\frac{1}{4} & R^{2}\left[-\cosh ^{2} \rho d t^{2}+d \rho^{2}+\sinh ^{2} \rho\left(d \eta^{2}+\sin ^{2} \eta d \chi^{2}\right)\right] \\
& +R^{2}\left[d \xi^{2}+\cos ^{2} \xi \sin ^{2} \xi\left(d \psi+\frac{1}{2} \cos \theta_{1} d \phi_{1}-\frac{1}{2} \cos \theta_{2} d \phi_{2}\right)^{2}\right. \\
& \left.+\frac{1}{4} \cos ^{2} \xi\left(d \theta_{1}^{2}+\sin ^{2} \theta_{1} d \phi_{1}^{2}\right)+\frac{1}{4} \sin ^{2} \xi\left(d \theta_{2}^{2}+\sin ^{2} \theta_{2} d \phi_{2}^{2}\right)\right] . \\
B_{\mathrm{NS}}=- & \frac{B}{2}\left(\sin 2 \xi \mathrm{d} \xi \wedge\left(2 \mathrm{~d} \psi+\cos \theta_{1} \mathrm{~d} \phi_{1}-\cos \theta_{2} \mathrm{~d} \phi_{2}\right)\right. \\
& \left.+\cos ^{2} \xi \sin \theta_{1} \mathrm{~d} \theta_{1} \wedge \mathrm{d} \phi_{1}+\sin ^{2} \xi \sin \theta_{2} \mathrm{~d} \theta_{2} \wedge \mathrm{d} \phi_{2}\right) .
\end{aligned}
$$

In addition to this there is a dilaton field and Ramond-Ramond two form and four form fields, whose detailed forms will not be needed in what follows. When taking $\alpha^{\prime}=1$, the curvature radius $R$ is given by $R^{2}=2^{5 / 2} \pi \lambda^{1 / 2}$, which is precisely same as that of ABJM. We are interested in a particular sector this model which can be obtained by choosing $\rho=0, \psi=$ constant and $\xi=\frac{\pi}{4}$ and by identifying that $\theta_{1}=\theta_{2} \equiv \theta$ and $\phi_{1}=\phi_{2} \equiv \phi$. With the above identifications, $\mathrm{AdS}_{4} \times \mathbf{C P}^{3}$ geometry reduces to $R \times S^{2}{ }^{1}$. The metric and the NS-NS flux reads

$$
\begin{aligned}
d s^{2} & =\frac{1}{4} R^{2}\left[-d t^{2}+\left(d \theta^{2}+\sin ^{2} \theta d \phi^{2}\right)\right], \\
B_{\mathrm{NS}} & =-\frac{B}{2}(\sin \theta \mathrm{d} \theta \wedge \mathrm{d} \phi) .
\end{aligned}
$$

\footnotetext{
${ }^{1}$ From the general equations of motion for the string moving inside the full $A d S_{4} \times \mathbf{C P}^{3}$, one can check that the above identification also gives a consistent solution
} 
We are interested in studying the classical rotating string around this geometry. We use the Polyakov action

$$
S=\frac{1}{4 \pi} \int d \sigma d \tau\left[\sqrt{-\gamma} \gamma^{\alpha \beta} g_{M N} \partial_{\alpha} x^{M} \partial_{\beta} x^{N}+e^{\alpha \beta} \partial_{\alpha} x^{M} \partial_{\beta} x^{N} B_{M N}\right]
$$

where we choose $\gamma^{\alpha \beta}=\eta^{\alpha \beta}$ as the world sheet metric and $e^{\alpha \beta}$ is the anti symmetric tensor defined as $e^{01}=-e^{10}=1$. In terms of target space coordinates the action is given by

$$
\begin{aligned}
S & =\frac{\pi \sqrt{2 \lambda}}{4 \pi} \int d \sigma d \tau\left[\left(\partial_{\tau} t\right)^{2}-\left(\partial_{\sigma} t\right)^{2}-\left(\partial_{\tau} \theta\right)^{2}+\left(\partial_{\sigma} \theta\right)^{2}-\sin ^{2} \theta\left\{\left(\partial_{\tau} \phi\right)^{2}-\left(\partial_{\sigma} \phi\right)^{2}\right\}\right] \\
& +\frac{B}{4 \pi} \int d \sigma d \tau \sin \theta\left[\left(\partial_{\sigma} \theta \partial_{\tau} \phi\right)-\left(\partial_{\tau} \theta \partial_{\sigma} \phi\right) .\right]
\end{aligned}
$$

To find the spiky string in this geometry, we choose the following parametrization

$$
t=f(\tau), \theta=\theta(y), \phi=\nu \tau+h(y)
$$

where $y=a \tau+b \sigma$ and we set $\tau$ and $\sigma$ run from $-\infty$ to $\infty$. Looking at the background geometry, one infers that there exist two conserved charges and the equations of motion for the corresponding fields are given by,

$$
\begin{aligned}
& 0=\partial_{\tau}^{2} f(\tau), \\
& 0=\partial_{y}\left[\sin ^{2} \theta\left\{a \nu+\left(a^{2}-b^{2}\right) h^{\prime}\right\}\right],
\end{aligned}
$$

where prime implies derivative with respect to $y$. The solutions of these equations are

$$
\begin{aligned}
f(\tau) & =\kappa \tau, \\
h^{\prime}(y) & =\frac{1}{a^{2}-b^{2}} \frac{c-a \nu \sin ^{2} \theta}{\sin ^{2} \theta},
\end{aligned}
$$

where $\kappa$ and $c$ are integration constants. Further the Virasoro constraints $T_{\alpha \beta}=0$ have also to be imposed. Due to the symmetry property of the metric, and the conformal nature of the Polyakov action the number of real independent constraints are two and we write them in the following form for our convenience

$$
T_{\tau \tau}+2 T_{\tau \sigma}=0, \quad T_{\tau \tau}-\frac{a^{2}+b^{2}}{2 a b} T_{\tau \sigma}=0
$$

The first Virasoro constraint gives rise to

$$
\theta^{\prime}=\frac{b \nu}{a^{2}-b^{2}} \frac{\sqrt{\left(\sin ^{2} \theta_{\max }-\sin ^{2} \theta\right)\left(\sin ^{2} \theta-\sin ^{2} \theta_{\min }\right)}}{\sin \theta},
$$

where

$$
\begin{aligned}
\sin ^{2} \theta_{\max }+\sin ^{2} \theta_{\min } & =\frac{\kappa^{2}(a-b)^{2}+2 b \nu c}{b^{2} \nu^{2}} \\
\sin ^{2} \theta_{\max } \cdot \sin ^{2} \theta_{\min } & =\frac{c^{2}}{b^{2} \nu^{2}} .
\end{aligned}
$$


The second Virasoro constraint is reduced to a relation among various parameters. From this we can set

$$
a=\frac{\nu}{\kappa^{2}} c .
$$

Note that in the above, the equation of motions are independent of NS-NS $B$ field. As previously mentioned, in this system there exists two conserved charges namely the total energy $E$ and total angular momentum $J$. The energy is given by

$$
\begin{aligned}
E & \equiv \frac{\sqrt{2 \lambda}}{2} \int d \sigma \partial_{\tau} t \\
& =\sqrt{2 \lambda} \int_{\theta_{\min }}^{\theta_{\max }} d \theta \frac{\kappa\left(a^{2}-b^{2}\right)}{b^{2} \nu} \frac{\sin \theta}{\sqrt{\left(\sin ^{2} \theta_{\max }-\sin ^{2} \theta\right)\left(\sin ^{2} \theta-\sin ^{2} \theta_{\min }\right)}}
\end{aligned}
$$

and angular momenta is given by

$$
\begin{aligned}
J & \equiv-\frac{\sqrt{2 \lambda}}{2} \int d \sigma \sin ^{2} \theta \partial_{\tau} \phi+\frac{B}{4 \pi} \int d \sigma \sin \theta \partial_{\sigma} \theta \\
& =-\sqrt{2 \lambda} \int_{\theta_{\text {min }}}^{\theta_{\max }} d \theta \frac{1}{b^{2} \nu} \frac{\sin \theta\left(a c-b^{2} \nu \sin ^{2} \theta\right)}{\sqrt{\left(\sin ^{2} \theta_{\max }-\sin ^{2} \theta\right)\left(\sin ^{2} \theta-\sin ^{2} \theta_{\min }\right)}}+\frac{B}{2 \pi} \int_{\theta_{\text {min }}}^{\theta_{\max }} d \theta \sin \theta .
\end{aligned}
$$

To consider a giant magnon or spike solution, we have to define the world sheet momentum $p$, which is identified with the angle difference $\Delta \phi \equiv p$,

$$
\begin{aligned}
\Delta \phi & \equiv-\int d \phi=-2 \int_{\theta_{\min }}^{\theta_{\max }} d \theta \frac{h^{\prime}}{\theta^{\prime}} \\
& =-2 \int_{\theta_{\min }}^{\theta_{\max }} d \theta \frac{1}{b^{2} \nu} \frac{\left(b c-a b \nu \sin ^{2} \theta\right)}{\sin \theta \sqrt{\left(\sin ^{2} \theta_{\max }-\sin ^{2} \theta\right)\left(\sin ^{2} \theta-\sin ^{2} \theta_{\min }\right)}}
\end{aligned}
$$

where we use a minus sign for making the angle difference positive.

\section{Giant Magnon and Single Spike Solutions}

In this section we will find the dispersion relation among various charges defined in the previous section, in the infinite size limit, which implies infinite angular momentum in case of giant magnon and infinite angle difference in case of a spiky string solution. This infinite size limit can be obtained by setting $\sin \theta_{\max }=1$ in both cases. In other words, this limit reduces Eq. (2.9) to

$$
\theta^{\prime}=\frac{b \nu}{a^{2}-b^{2}} \frac{\cos \theta \sqrt{\left(\sin ^{2} \theta-\sin ^{2} \theta_{\min }\right)}}{\sin \theta} .
$$

One sees that all of $E, J$ and $\Delta \phi$ diverge except for a special parameter region. In this limit, the second line in Eq. (2.10) gives $\sin ^{2} \theta_{\min }=\frac{c^{2}}{b^{2} \nu^{2}}$. The first line in Eq. (2.10) gives rise to a relation among various parameters. Using this relation and taking the help of Eq. (2.11), we get

$$
\kappa^{2}\left(a^{2}-b^{2}\right)=(c-b \nu)^{2}
$$




\section{Giant Magnon}

To find a solution which correspond to giant magnon, we first have to use a special parameter region which will make both $E-J$ and $\Delta \phi$ finite.

Below we summarize some details of identifying the parameter region. First we see that to cancel the logarithmic divergence in $E-J$ we get the following relation

$$
\kappa\left(a^{2}-b^{2}\right)=\left(a c-b^{2} \nu\right)
$$

If we further demand the finiteness of $\Delta \phi$, then we get

$$
c+2 a b \nu=0 .
$$

Using the relations among other parameters as mentioned in the previous section, we find that

$$
\nu=-\kappa, \quad a=-\frac{c}{\kappa}
$$

which makes

$$
\sin ^{2} \theta_{\min }=\frac{a^{2}}{b^{2}}
$$

With the above, one finds that making $E-J$ finite, $\Delta \phi$ automatically becomes finite and vice versa. The expression for $E-J$ and $\Delta \phi$ now becomes

$$
\begin{aligned}
E-J & =\left(\sqrt{2 \lambda}-\frac{B}{2 \pi}\right) z_{\max } \\
\Delta \phi & =2\left(\frac{\pi}{2}-\theta_{\min }\right)
\end{aligned}
$$

where $z_{\max }=\cos \theta_{\min }$. Finally we get the following dispersion relation for the giant magnon solution in the presence of NS-NS $B$ field as

$$
E-J=\left(\sqrt{2 \lambda}-\frac{B}{2 \pi}\right)\left|\sin \frac{\Delta \phi}{2}\right| .
$$

Note that for $B=0$, we get back the dispersion relation obtained in [10, 9].

\section{Single Spike}

In order to find a spike solution, we impose that $J$ is finite. We also impose that $E-\frac{\sqrt{2 \lambda}}{2} \Delta \phi$ is finite. So one finds following constraints on various parameters:

$$
a c=b^{2} \nu, \quad a=\frac{\nu}{\kappa^{2}} c,
$$

including those we have from the Virasoro constraints previously. A consistent parameter region can be summarized as follows

$$
a=-\frac{\nu}{\kappa}, \quad c=-\kappa, \quad \sin \theta_{\min }=\frac{\kappa}{\nu} .
$$


Finally one can compte the dispersion relation for the spike solution as

$$
\begin{gathered}
E-\frac{\sqrt{2 \lambda}}{2} \Delta \phi=\sqrt{2 \lambda} \bar{\theta} . \\
J=\left(\sqrt{2 \lambda}-\frac{B}{2 \pi}\right) \sin \bar{\theta}
\end{gathered}
$$

where $\bar{\theta}=\pi / 2-\theta_{\min }$. We further note that for $B=0$, the above relation reduces to that of [15].

\section{Finite Size effects}

We investigated giant magnon and spike solutions for the string in the infinite size limit in the last section.In this section we investigate the finite size effect on them. For that purpose we take $\theta_{\max } \neq \pi / 2$.

\section{Giant magnon:}

Eq. (2.10) and Eq. (2.11) gives

$$
\begin{aligned}
& \sin \theta_{\text {max }}=-\frac{\kappa}{\nu}, \\
& \sin \theta_{\text {min }}=\frac{c}{\kappa b},
\end{aligned}
$$

Assuming $z \equiv \cos \theta$, conserved charges are given by

$$
\begin{aligned}
E & =\sqrt{2 \lambda} \frac{z_{\max }^{2}-z_{\min }^{2}}{z_{\max } \sqrt{1-z_{\min }^{2}}} K(x), \\
J & =\sqrt{2 \lambda} z_{\max }[K(x)-E(x)]+\frac{B}{2 \pi}\left(z_{\max }-z_{\min }\right), \\
\frac{\Delta \phi}{2} & =\frac{\sqrt{1-z_{\min }^{2}}}{z_{\max } \sqrt{1-z_{\max }^{2}}} \Pi\left(\frac{z_{\max }^{2}-z_{\min }^{2}}{\sqrt{z_{\max }^{2}-1}} ; x\right)-\frac{\sqrt{1-z_{\max }^{2}}}{z_{\max } \sqrt{1-z_{\min }^{2}}} K(x),
\end{aligned}
$$

where we have used $z_{\text {max }}^{2} \equiv \cos ^{2} \theta_{\text {min }}=\frac{k^{2} b^{2}-c^{2}}{\kappa^{2} b^{2}}, z_{\min }^{2} \equiv \cos ^{2} \theta_{\max }=\frac{\nu^{2}-\kappa^{2}}{\nu^{2}}, x=\sqrt{1-\frac{z_{\min }^{2}}{z_{\max }^{2}}}$ and the elliptic integrals of the first, second and third kinds

$$
\begin{aligned}
K(x) & =\int_{z_{\min }}^{z_{\max }} d z \frac{z_{\max }}{\sqrt{\left(z_{\max }^{2}-z^{2}\right)\left(z^{2}-z_{\min }^{2}\right)}}, \\
E(x) & =\int_{z_{\min }}^{z_{\max }} d z \frac{z^{2}}{z_{\max } \sqrt{\left(z_{\max }^{2}-z^{2}\right)\left(z^{2}-z_{\min }^{2}\right)}} \\
\Pi\left(\frac{z_{\max }^{2}-z_{\min }^{2}}{\sqrt{z_{\max }^{2}-1}} ; x\right) & =\int_{z_{\min }}^{z_{\max }} d z \frac{z_{\max }\left(1-z_{\max }^{2}\right)}{\left(1-z^{2}\right) \sqrt{\left(z_{\max }^{2}-z^{2}\right)\left(z^{2}-z_{\min }^{2}\right)}} .
\end{aligned}
$$


To find the finite size effect, expanding conserved charges to $\mathcal{O}\left(z_{\min }^{2}\right)$ and $\mathcal{O}\left(z_{\max }^{2}\right)$, we obtain

$$
E-J \approx \sqrt{2 \lambda}\left(\left|\sin \frac{\Delta \phi}{2}\right|-\frac{z_{\max } z_{\min }^{2}}{4}\right)+\frac{B}{2 \pi}\left(z_{\min }-z_{\max }\right) .
$$

The leading behaviors of $E$ and $z_{\max }$ are given by

$$
\begin{aligned}
E & \approx \sqrt{2 \lambda} z_{\max } \log \frac{4 z_{\max }}{z_{\min }}, \\
z_{\max } & \approx\left|\sin \frac{\Delta \phi}{2}\right|, \\
z_{\min } & \approx\left|4 \sin \frac{\Delta \phi}{2}\right| e^{\left.-E /(\sqrt{2 \lambda}) \sin \frac{\Delta \phi}{2}\right)} .
\end{aligned}
$$

Using above relations, one obtains the following dispersion relation for giant magnon solution including the finite size correction as

$E-J=\sqrt{2 \lambda}\left(\left|\sin \frac{\Delta \phi}{2}\right|-4\left|\sin ^{3} \frac{\Delta \phi}{2}\right| e^{-E /\left(\sqrt{2 \lambda}\left|\sin \frac{\Delta \phi}{2}\right|\right)}\right)+\frac{B}{2 \pi}\left|4 \sin \frac{\Delta \phi}{2}\right|\left(e^{-E /\left(\sqrt{2 \lambda}\left|\sin \frac{\Delta \phi}{2}\right|\right)}-\frac{1}{4}\right)$.

For the infinite size case both $E$ and $J \rightarrow \infty$, which gives exactly same result obtained in the previous section.

\section{Single Spike:}

In this section we calculate finite size effect for the spike solution obtained in the previous section. Note that for the angular momentum to be positive in case of the spike solution, we should consider $J^{\prime} \equiv-J$ by changing the directions of rotation. Keeping this in mind, we now start to calculate the finite size effect for the spike.

Before calculating the dispersion relation, we should find $\sin \theta_{\min }$ and $\sin \theta_{\max }$, which are given by

$$
\begin{aligned}
& \sin \theta_{\min } \equiv \sqrt{1-z_{\max }^{2}}=\frac{\kappa}{\nu}, \\
& \sin \theta_{\max } \equiv \sqrt{1-z_{\min }^{2}}=\frac{c}{\kappa b} .
\end{aligned}
$$

With the above, the conserved charges can be rewritten as

$$
\begin{aligned}
E & =\sqrt{2 \lambda} \frac{z_{\max }^{2}-z_{\min }^{2}}{z_{\max } \sqrt{1-z_{\max }^{2}}} K(x), \\
J^{\prime} & =\sqrt{2 \lambda} \frac{1}{z_{\max }}\left(z_{\max }^{2} E(x)-z_{\min }^{2} K(x)\right)-\frac{B}{2 \pi}\left(z_{\max }-z_{\min }\right), \\
\frac{\Delta \phi}{2} & =\frac{\sqrt{1-z_{\min }^{2}}}{z_{\max } \sqrt{1-z_{\max }^{2}}}\left[K(x)-\Pi\left(\frac{z_{\max }^{2}-z_{\min }^{2}}{\sqrt{z_{\max }^{2}-1}} ; x\right)\right] .
\end{aligned}
$$

Here, the angular momentum $J^{\prime}$ is given by

$$
J^{\prime} \approx \sqrt{2 \lambda} z_{\max }-\left(\frac{1}{2}+\log \frac{4 z_{\max }}{z_{\min }}\right) \frac{\sqrt{2 \lambda} z_{\min }^{2}}{2 z_{\max }}-\frac{B}{2 \pi}\left(z_{\max }-z_{\min }\right),
$$


at $\mathcal{O}\left(z_{\text {min }}^{2}\right)$. Note that for the infinite size limit, $z_{\text {min }} \rightarrow 0$, the second term in the right hand side vanishes, so $J^{\prime}$ is always finite as it should do. The dispersion relation for a spike $E-\frac{\sqrt{2 \lambda}}{2} \Delta \phi$ is given by up to $\mathcal{O}\left(z_{\text {min }}^{3}\right)$ and $\mathcal{O}\left(z_{\text {max }}^{3}\right)$

$$
\begin{aligned}
E-\frac{\sqrt{2 \lambda}}{2} \Delta \phi & \approx \sqrt{2 \lambda} \arcsin z_{\max } \\
& -\left[\left(\frac{1}{2 z_{\max }}-\frac{z_{\max }}{4}\right) \frac{\sqrt{2 \lambda}}{2}+\left(\frac{1}{z_{\max }}+\frac{z_{\max }}{2}\right) \frac{\sqrt{2 \lambda}}{2} \log \frac{4 z_{\max }}{z_{\min }}\right] z_{\min }^{2} .
\end{aligned}
$$

To rewrite the dispersion relation in terms of the physical quantities, $z_{\min }$ and $z_{\max }$ should be replaced with $E$ and $J^{\prime}$. From the leading term of $E$ we obtain

$$
z_{\min }=4 z_{\max } e^{-E / \sqrt{2 \lambda} z_{\max }}
$$

and the leading term of $J^{\prime}$ gives

$$
z_{\max }=\frac{J^{\prime}}{\left(\sqrt{2 \lambda}-\frac{B}{2 \pi}\right)} \equiv \mathcal{J}(B) .
$$

So

$$
z_{\min }=4 z_{\max } e^{-E / \sqrt{2 \lambda} z_{\max }}=4 \mathcal{J}(B) e^{-E / \sqrt{2 \lambda} \mathcal{J}(B)}
$$

Using these, we finally obtain the dispersion relation for a finite size spike solution

$$
\begin{aligned}
E-\frac{\sqrt{2 \lambda}}{2} \Delta \phi & \approx \sqrt{2 \lambda} \arcsin \mathcal{J}(B) \\
& -8\left(\sqrt{1-(\mathcal{J}(B))^{2}} \frac{\sqrt{2 \lambda} \mathcal{J}(B)}{2}+\frac{E}{\sqrt{1-(\mathcal{J}(B)})^{2}}\right) e^{-2 E / \sqrt{2 \lambda} \mathcal{J}(B)}
\end{aligned}
$$

\section{Conclusions}

We have studied, in this paper, the rotating string in the diagonal $S U(2)$ subspace inside the $\mathrm{AdS}_{4} \times \mathbf{C P}^{3}$ geometry in the presence of a NS-NS $B$ field. We see that although the equations of motion of the rotating string on $R \times S^{2}$ are independent of NS-NS $B$ field, the most general form of conserved angular momentum depends on $B$ field. We have shown the existence of both the giant magnon, and the spike solutions for the string moving in this background which correspond to open spin chain and have found out the relevant dispersion relation among various charges in the infinite size limit. Furthermore, we have studied the finite size correction in both cases. It will be interesting to find more general string solutions in this background following [17]. 


\section{References}

[1] O. Aharony, O. Bergman, D.L. Jafferis and J. Maldacena, " $\mathcal{N}=6$ superconformal Chern-Simons-matter theories, M2-branes and their gravity duals," arXiv:0806.1218[hep-th]. JHEP 08 (2008) 001 [arXiv:0806.3391[hep-th]].

[2] O. Aharony, O. Bergman and D. L. Jafferis, "Fractional M2-branes," arXiv:0807.4924 [hep-th].

[3] J.M. Maldacena, "The large N limit of superconformal field theories and supergravity," Adv. Theor. Math. Phys. 2 (1998) 231 [arXiv:hep-th/9711200]; S.S. Gubser, I.R. Klebanov and A.M. Polyakov, "Gauge theory correlators from non-critical string theory," Phys. Lett. B428 (1998) 105 [arXiv:hep-th/9802109]; E. Witten, "Anti-de Sitter space and holography," Adv. Theor. Math. Phys. 2 (1998) 253 [arXiv:hep-th/9802150].

[4] D.M. Hofman and J.M. Maldacena, "Giant magnons," J. Phys. A39 (2006) 13095 [arXiv:hep-th/0604135].

[5] M. Kruczenski, JHEP 0508, 014 (2005) [arXiv:hep-th/0410226].

[6] M. Kruczenski, J. Russo and A.A. Tseytlin, J. High Energy Phys. 0610, 002 (2006) [arXiv:hep-th/0607044]. D76 (2007) 126006 [arXiv:0705.2429[hep-th]].

[7] M. Benna, I. Klebanov, T. Klose and M. Smedback, "Superconformal Chern-Simons theories and $A d S_{4} / C F T_{3}$ correspondence," JHEP 09 (2008) 072 [arXiv:0806.1519[hep-th]].

[8] J.A. Minahan and K. Zarembo, "The Bethe ansatz for superconformal Chern-Simons," JHEP 09 (2008) 040 [arXiv:0806.3951[hep-th]].

[9] D. Gaiotto, S. Giombi and X. Yin, "Spin chains in $\mathcal{N}=6$ superconformal Chern-Simons-matter theory," arXiv:0806.4589[hep-th].

[10] G. Grignani, T. Harmark and M. Orselli, "The $S U(2) \times S U(2)$ sector in the string dual of $\mathcal{N}=6$ superconformal Chern-Simons theory," arXiv:0806.4959[hep-th].

[11] D. Bak and S. J. Rey, "Integrable Spin Chain in Superconformal Chern-Simons Theory," JHEP 0810, 053 (2008) [arXiv:0807.2063 [hep-th]].

[12] G. Arutyunov and S. Frolov, "Superstrings on $A d S_{4} \times C P^{3}$ as a coset sigma-model," arXiv:0806.4940[hep-th]; B.J. Stefanski, "Green-Schwarz action for type IIA strings on $A d S_{4} \times C P^{3}$," arXiv:0806.4948[hep-th]; G. Bonelli, P. A. Grassi and H. Safaai, "Exploring Pure Spinor String Theory on AdS(4) X CP**3," arXiv:0808.1051 [hep-th].

[13] N. Gromov and P. Vieira, "The all loop AdS4/CFT3 Bethe ansatz," arXiv:0807.0777 [hep-th].

[14] N. Gromov and P. Vieira, "The $A d S_{4} / C F T_{3}$ algebraic curve," arXiv:0807.0437[hep-th].

[15] B.H. Lee, K.L. Panigrahi and C. Park, "Spiky strings on $A d S_{4} \times C P^{3}$," arXiv:0807.2559[hep-th].

[16] B. Chen and J.B. Wu, "Semi-classical strings in $A d S_{4} \times C P^{3}$," arXiv:0807.0802[hep-th].

[17] Shijong Ryang, "Giant Magnon and Spike Solutions with Two Spins in AdS4xCP3," arXiv:0809.5106 [hep-th].

[18] C. Ahn, P. Bozhilov and R.C. Rashkov, "Neumann-Rosochatius integrable system for strings on $A d S_{4} \times C P^{3}$," JHEP 09 (2008) 017 [arXiv:0807.3134[hep-th]]. 
[19] I. Shenderovich, "Giant magnons in $A d S_{4} / C F T_{3}$ : dispersion, quantization and finite-size corrections," arXiv:0807.2861[hep-th].

[20] T. McLoughlin and R. Roiban, "Spinning strings at one-loop in $A d S_{4} \times P^{3}$," arXiv:0807.3965[hep-th].

[21] L.F. Alday, G. Arutyunov and D. Bykov, "Semiclassical quantization of spinning strings in $A d S_{4} \times C P^{3}, "$ arXiv:0807.4400[hep-th].

[22] C. Krishnan, "AdS $S_{4} / C F T_{3}$ at one loop," arXiv:0807.4561[hep-th].

[23] N. Gromov and V. Mikhaylov, "Comment of the scaling function in $A d S_{4} \times C P^{3}$," arXiv:0807.4897[hep-th].

[24] D. Bak, D. Gang and S.J. Rey, "Integrable spin chain of superconformal $U(M) \times \bar{U}(N)$ Chern-Simons theory," arXiv:0808.0170[hep-th].

[25] R. Ishizeki and M. Kruczenski, "Single spike solutions for strings on $S^{2}$ and $S^{3}$," Phys. Rev.

[26] N.P. Bobev and R.C. Rashkov, "Spiky strings, giant magnons and beta-deformations," Phys. Rev. D76 (2007) 046008 [arXiv:0706.0442[hep-th]]; Hayashi, K. Okamura, R. Suzuki and B. Vicedo, "Large winding sector of AdS/CFT," JHEP 11 (2007) 033 [arXiv:0709.4033[hep-th]]; H. Dimov and R.C. Rashkov, "On the anatomy of multi-spin magnon and single spike string solutions," Nucl. Phys. B799 (2008) 255 [arXiv:0709.4231[hep-th]].

[27] J. Kluson, R.R. Nayak and K.L. Panigrahi, "Giant magnon in NS5-brane background," JHEP 04 (2007) 099 [arXiv:hep-th/0703244];B.H. Lee, R.R. Nayak, K.L. Panigrahi and C. Park, "On the giant magnon and spike solutions for strings on $A d S_{3} \times S^{3}$," JHEP $06(2008)$ 065 [arXiv:0804.2923[hep-th]]; J.R. David and B. Sahoo, "Giant magnons in the D1-D5 system," JHEP 07 (2008) 033 [arXiv:0804.3267[hep-th]]; J. Kluson, B.H. Lee, K.L. Panigrahi and C. Park, "Magnon like solutions for strings in I-brane background," JHEP 08 (2008) 032 [arXiv:0806.3879[hep-th]]; C.M. Chen, J.H. Tsai and W.Y. Wen, "Giant magnons and spiky strings on $S^{3}$ with B-field," arXiv:0809.3269[hep-th];J. Kluson,K.L. Panigrahi, "D1-brane in beta-Deformed Background," JHEP 0711:011,(2007) arXiv:0710.0148 [hep-th].

[28] G. Arutyunov, S. Frolov and M. Zamaklar, "Finite-size effects from giant magnons," Nucl. Phys. B778 (2007) 1 [arXiv:hep-th/0606126];D. Astolfi, V. Forini, G. Grignani and G.W. Semenoff, "Gauge invariant finite size spectrum of the giant magnon," Phys. Lett. B651 (2007) 329 [arXiv:hep-th/0702043]; Y. Hatsuda and R. Suzuki, "Finite-size effects for dyonic giant magnons," Nucl. Phys. B800 (2008) 349 [arXiv:0801.0747[hep-th]]; "Finite-size effects for multi-magnon states," arXiv:0807.0643[hep-th];C. Ahn and P. Bozhilov, "Finite-size effects for single spike," JHEP 07 (2008) 105 [arXiv:0806.1085[hep-th]]; T. Klose and T. McLoughlin, "Interacting finite-size magnons," J. Phys. A41 (2008) 285401 [arXiv:0803.2324[hep-th]]; B. Ramadanovic and G.W.Semenoff, "Finite size giant magnon," arXiv:0803.4028[hep-th];G. Grignani, T. Harmark, M. Orselli and G.W. Semenoff, "Finite size giant magnons in the string dual of $\mathcal{N}=6$ superconformal Chern-Simons theory," arXiv:0807.0205[hep-th]; D. Astolfi, V.G.M. Puletti, G. Grignani, T. Harmark and M. Orselli, "Finite-size corrections in the $S U(2) \times S U(2)$ sector of type IIA string theory on $A d S_{4} \times C P^{3}$," arXiv:0807.1527[hep-th]. Changrim Ahn, P. Bozhilov, " Finite-size Effect of the Dyonic Giant Magnons in N=6 super Chern-Simons Theory," arXiv:0810.2079 [hep-th];Tomasz Lukowski, Olof Ohlsson Sax, "Finite size giant magnons in the $S U(2) x S U(2)$ sector of $A d S_{4} x C P^{3}$, " arXiv:0810.1246 [hep-th];Diego Bombardelli, Davide Fioravanti, "Finite-Size Corrections of the $C P^{3}$ Giant Magnons: the Luscher terms," 
arXiv:0810.0704 [hep-th]; J. A. Minahan and O. Ohlsson Sax, "Finite size effects for giant magnons on physical strings", Nucl. Phys. B801 (2008) 97 [arXiv:0801.2064 [hep-th]];

N. Gromov, S. Schafer-Nameki and P. Vieira, "Quantum Wrapped Giant Magnon," Phys. Rev. D 78 (2008) 026006 [arXiv:0801.3671 [hep-th]]; N. Gromov, S. Schafer-Nameki and P. Vieira, "Efficient precision quantization in AdS/CFT," arXiv:0807.4752 [hep-th]. 